Instituto Internacional de Investigación y Desarrollo Tecnológico Educativo INDTEC, C.A.

DOI: https://doi.org/10.29394/Scientific.issn.2542-2987.2021.6.21.5.101-123

OAl-PMH: http://www.indteca.com/ojs/index.php/Revista Scientific/oai

\title{
El refuerzo académico en experiencias de aprendizaje para el Ámbito de relaciones lógico-matemáticas con GeoGebra
}

\author{
Autoras: Diana Isabel Rodríguez Rodríguez \\ Universidad Nacional de Educación, UNAE \\ diana.rodriguez@unae.edu.ec \\ Azogues, Ecuador \\ https://orcid.org/0000-0003-4406-9555 \\ Charly Marlene Valarezo Encalada \\ Universidad Nacional de Educación, UNAE \\ charly.valarezo@unae.edu.ec \\ Azogues, Ecuador \\ https://orcid.org/0000-0002-6540-8395 \\ Dennise Adriana Velecela Cordero \\ Universidad Nacional de Educación, UNAE \\ davelecela@unae.edu.ec \\ Azogues, Ecuador \\ https://orcid.org/0000-0002-3475-0235
}

\section{Resumen}

El presente artículo comparte las experiencias desarrolladas en el proyecto de vinculación Rincón Infantil de Aprendizaje Móvil Social (RIAMOS), de la Universidad Nacional de Educación (UNAE), Ecuador. En el ciclo IIS-2020, en la modalidad virtual una de las Aristas, la de Aproximación a la Geometría y Medida, cuyo objetivo fue aplicar GeoGebra como una herramienta digital en el ámbito de relaciones lógico-matemáticas en la Educación Inicial (EI), partiendo de las necesidades detectadas en los niños y niñas mediante una evaluación diagnóstica. Durante este proceso se realizó capacitaciones, elaboración de instrumentos, creación de actividades que responden al Currículo Nacional de El, implementación, sistematización y socialización de resultados con base en la descripción cualitativa e interpretativa de la información obtenida. Se demuestra que, gracias a los promotores educativos, se fortaleció con refuerzo académico el área abordada. Además, se proporciona insumos de buenas prácticas que mediante la reflexión deben continuar para el desempeño de nuevas acciones innovadoras en la práctica docente.

Palabras clave: necesidades educacionales; práctica pedagógica; docencia.

Código de clasificación internacional: 5802.04 - Niveles y temas de educación.

\begin{abstract}
Cómo citar este artículo:
Rodríguez, D., Valarezo, C., \& Velecela, D. (2021). El refuerzo académico en experiencias de aprendizaje para el Ámbito de relaciones lógico-matemáticas con GeoGebra. Revista Scientific, 6(21), 101-123, e-ISSN: 2542-2987. Recuperado de: https://doi.org/10.29394/Scientific.issn.2542$\underline{2987.2021 .6 .21 .5 .101-123}$
\end{abstract}

Fecha de Recepción: 12-05-2021
Fecha de Aceptación: 03-07-2021
Fecha de Publicación: 05-08-2021 
OAl-PMH: http://www.indteca.com/ojs/index.php/Revista Scientific/oai

Artículo Original / Original Article

\title{
Academic reinforcement in learning experiences for the Field of logical- mathematical relationships with GeoGebra
}

\begin{abstract}
This article shares the experiences developed in the Social Mobile Learning Children's Corner project (RIAMOS), of the National University of Education (UNAE), Ecuador. In the IIS-2020 cycle, in the virtual modality one of the Edges, the Approach to Geometry and Measurement, whose objective was to apply GeoGebra as a digital tool in the field of logical-mathematical relationships in Initial Education (IE), starting from the needs detected in the boys and girls through a diagnostic evaluation. During this process, training was carried out, elaboration of instruments, creation of activities that respond to the National El Curriculum, implementation, systematization and socialization of results based on the qualitative and interpretive description of the information obtained. It is shown that, thanks to the educational promoters, the area addressed was strengthened with academic reinforcement. In addition, it provides inputs of good practices that through reflection should continue for the performance of new innovative actions in teaching practice.
\end{abstract}

Keywords: educational needs; pedagogical practice; teaching. International classification code: 5802.04 - Levels and subjects of education.

How to cite this article:

Rodríguez, D., Valarezo, C., \& Velecela, D. (2021). Academic reinforcement in learning experiences for the Field of logical-mathematical relationships with GeoGebra. Revista Scientific, 6(21), 101-123, e-ISSN: 2542-2987. Recovered from: https://doi.org/10.29394/Scientific.issn.2542-2987.2021.6.21.5.101-123

Date Received: 12-05-2021
Date Acceptance:

03-07-2021
Date Publication: 05-08-2021 


\section{Introducción}

Este artículo científico aborda las necesidades detectadas en las experiencias de aprendizaje para el desarrollo del pensamiento lógico matemático con GeoGebra en Educación Inicial (EI) mediante el refuerzo académico. La herramienta antes mencionada es un apoyo fundamental en las clases virtuales, pues, al ser bastante intuitiva permite guiar la ejecución de las actividades a los niños y niñas con el fin de alcanzar dominio en las destrezas con criterio de desempeño en este ámbito de desarrollo y aprendizaje. Con esto se propicia una alternativa pedagógica dinámica y motivante de aprendizaje en esta modalidad, en la que León y Cisneros (2021): manifiestan que "[...] los profesores utilicen diferentes metodologías y compartan con sus alumnos diversos recursos digitales para aprovechar al máximo la tecnología y así evitar que las aulas virtuales se conviertan en espacios a los que se han trasladado las viejas prácticas [...]" (pág. 99).

En esta misma línea, el nexo cognitivo que se crea a través del pensamiento lógico matemático y las actividades planteadas resulta en la concreción de aprendizajes significativos resaltados en los postulados del Currículo de El y en el fortalecimiento de necesidades específicas mediante el refuerzo académico.

Con el abordaje de la relación número cantidad; clasificación objetos con un atributo (tamaño, color o forma); reconocimiento y comparación de objetos de acuerdo con su tamaño grande, mediano, pequeño; y la identificación de figuras geométricas básicas, mediante un análisis interpretativo de las actividades desarrolladas en las intervenciones. Se concluye mencionando que el refuerzo académico es una estrategia de valor en la práctica docente en la modalidad virtual. En efecto, el objetivo de la presente investigación radica en aplicar GeoGebra como una herramienta digital en el ámbito de relaciones lógico-matemáticas en El. 


\section{Metodología (Materiales y métodos)}

El presente trabajo investigativo es de carácter cualitativo con aplicación del método interpretativo, puesto que para Flick (2015): reflexionar sobre la percepción del propio individuo se construye con experiencias ante una situación, contexto estudio de caso, siendo capaz de interpretar y valorar sus acciones para decidir su forma de actuar. Asimismo, la sistematización de experiencias direccionó la obtención de los resultados relacionados a la concepción de las buenas prácticas del docente en el proceso de enseñanza.

La población estuvo conformada por 22 estudiantes de la carrera de Educación Inicial (EI) de la Universidad Nacional de Educación (UNAE), y por 55 niños y niñas de instituciones educativas y comunidades de las provincias de Azuay y Cañar.

Se partió de una encuesta inicial dirigida a las familias de los infantes involucrados, posterior a ello, un diagnóstico aplicado a los educandos, seguido de una propuesta de intervención educativa, una post evaluación y una encuesta de satisfacción. Todo este proceso mediante actividades diseñadas en la herramienta de GeoGebra, que se fundamentan en las destrezas del Ámbito de Relaciones Lógico-Matemáticas del Subnivel Inicial II del sistema educativo ecuatoriano.

Todas las sesiones desde su inicio fueron individualizadas para cada niño y niña, guiadas por los estudiantes, quienes a su vez eran los responsables de registrar todos los eventos suscitados durante el acompañamiento; también se vio el involucramiento de la familia en este proceso, considerando que en algunas ocasiones los infantes requerían de su apoyo para el manejo de la herramienta de GeoGebra.

\subsection{Refuerzo académico}

Desde nuestra experiencia entendemos que el refuerzo académico es el apoyo pedagógico que brindan los docentes a los educandos. Para un mejor 
entendimiento de esto, es necesario tener un acercamiento al concepto y las particularidades propias de este, es así que para Longás, Civís y Riera (2013): es "[...] un conjunto de estrategias y acciones que tienen por finalidad mejorar los procesos de aprendizaje, inclusión y éxito escolar [...]” (pág. 109). Entendiéndose como a la puesta en marcha de una programación de soporte al proceso de aprendizaje orientada a aquellos estudiantes que requieren atención personalizada.

Sumando a lo anterior, Herberth (2015): menciona que "[...] el refuerzo académico se vuelve necesario en la medida en que la institución educativa no logra que el estudiante obtenga la eficacia en el rendimiento académico" (pág. 13). Y que, a su vez se da debido a que muchas veces en casa las familias no están preparadas pedagógicamente para solventar las necesidades académicas de los estudiantes. Pudiendo darse durante la jornada educativa o también en horas complementarias fuera del horario académico, en donde las actividades que se realicen deben apuntar a la adquisición de aprendizajes significativos teniendo como base un modelo constructivista.

Al lado de ello, la intervención de los actores educativos (directivos, docentes, padres de familia y/o representantes legales) en este proceso es fundamental y estos deben tener en cuenta los elementos y acciones para el desarrollo del refuerzo académico determinados en el Instructivo para la aplicación de la evaluación estudiantil. Por otro lado, también se recomienda que las estrategias metodológicas asumidas por el docente/tutor se ajusten al contexto, cambios actuales, intereses, capacidades y necesidades de los estudiantes teniendo en cuenta los estilos y ritmos de aprendizaje de cada uno.

En ese sentido, el Ministerio de Educación del Ecuador (2017), citado por Ortega y Taquez (2019): propone el “[...] refuerzo académico individualizado, refuerzo académico grupal, ayuda entre iguales, aprendizaje a través del uso de la tecnología, aprendizaje cooperativo, enseñanza 
compartida y tareas escolares para la casa" (págs. 21-22). Cada una de estas con sus propias características y niveles de dificultad, siendo el docente el responsable del diseño e implementación del plan de recuperación, que dependerá de las particularidades de los casos.

El papel del docente, además, está en encontrar una metodología adecuada que atraiga el interés de los educandos, y que para los infantes se pueda ejecutar mediante los ambientes de aprendizaje adecuados, que conlleve al progreso en la adquisición de destrezas de los ámbitos de desarrollo.

\section{2. Ámbito de desarrollo de la Lógico - Matemática}

Partiendo desde el estudio de Padilla (2018): en el que se señala que "la inteligencia lógico-matemática conlleva numerosos componentes: cálculos matemáticos, pensamiento lógico, resolución de problemas y razonamiento deductivo e inductivo" (pág. 171); se determina que las matemáticas en la primera infancia se relacionan a ese pensamiento lógico, espacial, temporal, causal y de medidas.

En efecto, el pensamiento lógico infantil se consolida en las dos primeras etapas del desarrollo cognitivo descritas por Piaget (1961): la sensoriomotriz y la preoperacional, centrados en el aprendizaje mediante los sentidos y el desarrollo de la función simbólica. La variabilidad de experiencias que el niño adquiere en consonancia consigo mismo, con los demás y con los objetos que lo rodean, transfieren ideas a su mente sobre las relaciones entre causa y efecto.

Como producto de esa internalización se da pasó al conocimiento que, contrastadas con anteriores y nuevas experiencias, los infantes comprenden el sentido de lo correcto y lo incorrecto. De tal manera que para Reyes-Vélez (2017): "la interpretación del conocimiento matemático [...] se construye mediante una dinámica de relaciones, sobre la cantidad y la posición de los 
objetos en el espacio y en el tiempo" (pág. 200).

Por lo tanto, el desarrollo del pensamiento lógico matemático se da a partir de las experiencias y de las interacciones del niño con el entorno, es decir, desde la formación de su autoconcepto físico, que se da a partir de lo que el infante reconoce como cambios en situaciones sencillas y cotidianas, debido a que caracteriza y establece relaciones entre los objetos y realiza acciones. En suma, los aprendizajes matemáticos se conjugan a partir de la estructuración del esquema corporal, y de la conciencia de los conceptos tales como: nociones de objeto, ubicación en el tiempo y en el espacio, y nociones de orden.

El Currículo de Educación Inicial publicado por el Ministerio de Educación del Ecuador (MINEDUC, 2014a): en el ámbito de relaciones lógicomatemáticas "comprende el desarrollo de los procesos cognitivos con los que el niño explora y comprende su entorno y actúa sobre él para potenciar los diferentes aspectos del pensamiento" (pág. 32); mediante este ámbito además de las "[...] nociones básicas de tiempo, cantidad, espacio, textura, forma, tamaño y color [...]" (pág. 32); se propicia la adquisición de las operaciones sustantivas que se construyen de manera simultánea y que constituyen la base para el desarrollo cognitivo en los niños, a partir de los procesos de clasificación, seriación y correspondencia.

Estas operaciones lógicas esenciales en la primera infancia se deben trabajar mediante la aplicación de didácticas lúdicas que permitan al niño la asimilación total de la realidad para comprenderla y aprovecharla. Generalmente el infante disfruta mucho los juegos independientemente del contexto en que se encuentren, y estos acompañados de la tecnología hoy en día son importantes en su formación.

En este sentido, Torres (2010): propone que las generaciones que en los tiempos actuales están naciendo y creciendo, se educan en un contexto tecnológico avanzado y globalizado. Por lo tanto, muestran más posibilidades 
como estudiantes para desarrollar habilidades, capacidades y talento. Sumado a esto el acompañamiento del docente para el niño, posibilitará descubrir el placer y la utilidad de las matemáticas para comprender el mundo.

\subsection{La herramienta de GeoGebra en la Educación Inicial}

Para la educación inicial GeoGebra constituye una herramienta innovadora que aproxima al niño a las nociones básicas y operaciones sustanciales de la geometría y medida, construyendo conocimientos según sus necesidades cognitivas. Pues tal como menciona Carrillo (2012), citado por Rizzo y Costa (2019): "su utilización en el aula de matemática es imprescindible, porque permite realizar construcciones que en lápiz y papel no podrían estar realizándose" (pág. 3).

En suma, GeoGebra como herramienta de apoyo al docente de educación inicial, favorece el desarrollo del pensamiento lógico-matemático en el educando, porque facilita su movilización entre los sistemas de representación simbólica. Para Calvo (2008), citado por Rojas-Bello (2020): es en este proceso que el docente busca el vínculo entre la nueva formación con el entendimiento que ya posee el estudiante, aprovechando al mismo tiempo el fomento de la autoconfianza al reconocer que la información previa que posee, es muy importante para la ruta de enseñanza.

\section{Resultados (análisis e interpretación de los resultados)}

El proyecto de vinculación RIAMOS, en aras de propiciar un apoyo a la comunidad en general implementó un macroproyecto, derivando aristas, una de ellas es de Aproximación a la Geometría y Medida con la herramienta de GeoGebra. A partir, de él se abordó temáticas del ámbito de Relaciones lógicomatemáticas, con el propósito de realizar un refuerzo académico desde la asignatura. De esta manera, se propició un trabajo conjunto con las estudiantes (promotoras educativas del proyecto), en la detección de 
necesidades presentadas en las prácticas preprofesionales, así como también en el contexto de los estudiantes con horas de vinculación.

Con base en el proyecto se desarrolló actividades con el apoyo de la herramienta de GeoGebra que propició el apoyo a los niños con necesidades. Se consideró algunos aspectos relevantes que incidieron significativamente en la ejecución como la capacitación en la herramienta, el espacio virtual o físico para la atención a los niños. Sin embargo, por la relevancia de la aplicación los resultados en general fueron óptimos de ser compartidos y publicados en un espacio adecuado.

\subsection{Intención del refuerzo académico}

Uno de los aspectos analizados en la recolección de información es el rol que cumple el refuerzo académico, como parte activa en los centros educativos para el mejoramiento del rendimiento escolar de los niños. Sin embargo, al hablar de rendimiento nos enfocamos en el desarrollo de destrezas y habilidades más no en una mera práctica evaluativa. Señalando a Edel (2003): sobre los procesos evaluativos, se considera al rendimiento como calidad educativa, en el sentido de un cambio profundo y abstracto para fortalecer sus aprendizajes futuros. Una de las propuestas en el currículo del MINEDUC (2014b): es "la formación integral de los niños, esto implica el desarrollo de los diferentes ámbitos que permiten especificar la tridimensionalidad de la formación del ser humano, es decir, lo actitudinal, lo cognitivo y lo psicomotriz [...]" (pág. 17).

Así, en el análisis de la información obtenida desde las prácticas desarrolladas, a través del proyecto se considera aspectos relevantes entorno a las necesidades detectadas como las dificultades para reconocer los colores y las figuras geométricas en la mayoría de los casos. Pues, se determinó cuando el niño dudaba de su respuesta o simplemente lanzaba respuestas al azar. En efecto, en las primeras sesiones por ejemplo debían reconocer las 
figuras geométricas básicas y sucedió que, conocían los nombres más no podían identificar cual era el cuadrado o el triángulo.

Con base en las limitaciones se realizó planificaciones entorno a actividades como parte del refuerzo académico con el apoyo de la herramienta de GeoGebra. Donde, como descripción se tenía que ordenar las figuras presentadas según lo patrones (grande, mediano y pequeño). También se abordó las nociones espaciales de una figura con respecto a otra. Dichas actividades ayudaron a fortalecer algunas destrezas, con la recomendación de trabajar con objetos del contexto para el fortalecimiento.

En este sentido, GeoGebra se aplicó mediante la construcción del Tangram para reforzar las figuras geométricas que ya conocían. Se obtuvo resultados alentadores, pues este recurso junto a sus bondades apoyo significativamente a la experiencia de aprendizaje. Se trabajó lateralidad (izquierda - derecha), relación número-cantidad y conteo oral.

\subsection{El refuerzo académico en el Ámbito de las Relaciones lógico- matemáticas}

Uno de los aspectos que se consideró en el refuerzo académico es el ritmo de aprendizaje, considerando algunos aspectos como la motivación que el niño y la niña presentan y con base en la velocidad en la que el niño pueda adquirir cierto conocimiento. Como se menciona en el Currículo del MINEDUC (2014c): respecto a las diferencias individuales, se menciona la relevancia del manejo de la flexibilidad en la práctica docente toma en consideración las formas de aprender individualizadas. Conjuntamente, con el fomento de estrategias didácticas innovadoras y creativas que desarrollen a cabalidad las destrezas y habilidades respetando la heterogeneidad.

Otro de los aspectos relevantes en el refuerzo académico es la retroalimentación para generar conciencia de los aciertos y desaciertos en una experiencia de aprendizaje. Esto propicia una estimulación personalizada y 
profunda que contribuye en la oportunidad de mejorar la comprensión al considerar temas ya tratados.

El ámbito de relaciones lógico-matemáticas es una de las asignaturas que se desarrollan desde los 3 años de edad en educación inicial. Se considera las competencias relacionadas con el número, forma, espacio y medida. Donde, las estrategias aplicadas constituyen un elemento primordial en el desenvolvimiento del niño y de la niña.

Con base en ello, se manifiesta que la competencia relacionada con el número aborda operaciones básicas aritméticas, agrupar objetos por sus atributos cualitativos y cuantitativos. Para Cardoso y Cerecedo (2008a): se identifica la secuencia con criterios de repetición y crecimiento, se trabaja los principios de conteo identificando la cantidad por estimación de objetos, plantea y resuelve situaciones cotidianas que implican agregar, reunir, quitar, igualar, comparar y compartir.

En relación con el desarrollo de la forma, espacio y medida se menciona en Cardoso y Cerecedo (2008b): quienes nombran las características de objetos, figuras y cuerpos geométricos, empleando un lenguaje convencional para reconocer y presentarlas en diferentes perspectivas. Se construye sistemas de referencia con relación a la ubicación espacial, utilizando términos como dentro, fuera, arriba, abajo, encima, cerca, lejos. Al mismo tiempo, se utilizan unidades no convencionales para resolver problemas que implican medir magnitudes de longitud, capacidad, peso, tiempo. Así, se determina la utilidad de los instrumentos de medición.

Para la aplicación, se realizaron planificaciones sustentadas en el Currículo de Educación Inicial del MINEDUC (2014d): donde se consideraron las destrezas dependiendo de la edad de los niños y niñas. Se ejercitó en el conteo oral del 1 al 15 con secuencia numérica. Se identifica características del día y la noche, con sus particularidades y el apoyo de los acontecimientos, se ordena en secuencias lógica sucesos de hasta tres eventos, en actividades 
de la rutina diaria en escenas de cuentos con representaciones gráficas. Se reconoce ubicación espacial con relación al niño en su contexto, arriba/ abajo, al lado, dentro/fuera, cerca/lejos, adelante/atrás, junto a.

Se continúa con la clasificación de objetos con uno y dos atributos (tamaño, color o forma). Adicional a ello, se escribe formas básicas círculo, triángulo, rectángulo y cuadrado en objetos del entorno y con el apoyo de la herramienta de GeoGebra.

\subsection{El refuerzo académico en el Ámbito de las Relaciones lógico- matemáticas con GeoGebra}

El desarrollo del pensamiento lógico en la temprana edad gira en torno a la adecuada estimulación que se realiza en la práctica docente. Así, el desarrollo de la inteligencia se da de manera natural y sin esfuerzo, a partir de las destrezas y habilidades adquiridas según las experiencias diarias vividas.

Respecto a este enunciado se pude mencionar que las estrategias juegan un papel primordial en la práctica adecuada. La búsqueda de herramientas que proporcionen estos beneficios debe ser bien pensadas. También, discurrir aspectos que aborden la virtualidad por la pandemia que se atraviesa. De esta manera, GeoGebra con sus bondades proporciona múltiples alternativas para el desarrollo del pensamiento lógico matemático. Torres (2014): alude que, al cambiar las metodologías tradicionales usadas por los docentes, se reemplaza por las experiencias de aprendizaje adquiridas con GeoGebra se tornan significativas a medida que se desarrollan en los refuerzos.

Pues, al impartir conocimiento de la herramienta aporta a las necesidades educativas del niño y de la niña, a través del diseño de actividades y la variedad de elementos que posee. Entre ellos son los colores, formas, figuras que llaman la atención de los niños y niñas, y estimulan el desarrollo de destrezas y habilidades, mediante el juego. 
Asimismo, la interacción entre el facilitador y el niño y la niña en las clases en línea es óptimo, constituyéndose en un recurso didáctico de calidad. Con la aplicación de un sinnúmero de actividades, dependiendo de la creatividad y el objetivo educativo, el docente pone en juego sus habilidades para transmitir un conocimiento visual para aprender de manera divertida, dinámica y adentrarse en la tecnología.

Actualmente, surge como una necesidad la utilización de herramientas tecnológicas más aún, por la emergencia sanitaria que estamos atravesando en el mundo. Por lo tanto, GeoGebra es un método de enseñanza que permite estimular la concentración en el niño y la niña para generar los conocimientos que se requieren.

En la planificación de las actividades con la herramienta, se centró la utilización de elementos llamativos que provoquen un aprendizaje activo por medio de colores, figuras, imágenes que aportaron al desarrollo de destrezas. Se consideró a la herramienta como innovadora en la etapa de inicial, pues en algunos casos es la primera vez que se utilizó, generando expectativa y motivación por aprender.

Por ejemplo, la aplicación de actividades lúdicas con el apoyo del Tangram, la animación y las figuras en 3D en GeoGebra, propiciando nuevas alternativas de aprendizaje, lo que conllevó a determinar que el refuerzo académico fue un éxito. De hecho, se puede manifestar que se pudo fortalecer varias destrezas a la vez por ser una herramienta dinámica y sugestiva que permite trabajar interactiva e idóneamente en las experiencias de aprendizaje.

Con base en la aplicación de GeoGebra se menciona en los instrumentos de recolección final, que esta, cumple con las expectativas para abordar el ámbito de relaciones lógico-matemáticas. Se sitúa como una opción intuitiva dentro del mundo de las Tecnologías de la Información y la Comunicación (TIC), sobre todo se resalta la innecesaridad del uso del internet, y que principalmente aporta de manera significativa a la transmisión 
de conocimientos en la primera infancia. Igualmente, se proyecta a la educación presencial como una alternativa innovadora y de amplias posibilidades en su utilización como estrategia didáctica dentro del aula.

En otras palabras, se busca alternativas que mejoren los espacios de aprendizaje con nuevas iniciativas didácticas, que dejen de lado la manera tradicional de enseñar. Se incentiva a sumergirse en el desarrollo computacional en el proceso de enseñanza- aprendizaje. Propendiendo de reflexiones en las prácticas docentes que propicien la mejora continua en las experiencias de aprendizaje.

\subsection{Buenas prácticas con GeoGebra}

En las buenas prácticas con la herramienta de GeoGebra se logra comprender el sentido y el significado en los niños y niñas a temprana edad. En efecto, se deben desarrollar con base a experiencias de aprendizaje de calidad y calidez, partiendo de la observación y el movimiento de objetos contextualizados.

Así, mediante la planificación de actividades innovadoras en el ámbito de relaciones lógico-matemáticas se logra un enfoque en el aprendizaje y la atención de los niños. Donde, se obtiene nuevas experiencias de aprendizaje significativas y creativas que aportan a la construcción de su conocimiento.

En consecuencia, Marqués (2002), citado por Jorquera (2017): define como buenas prácticas "las intervenciones educativas que facilitan el desarrollo de actividades de aprendizaje en las que se logren con eficiencia los objetivos formativos previstos y también otros aprendizajes de alto valor educativo" (pág. 74). Entonces, al hablar de buenas prácticas se hace referencia a todas aquellas experiencias o intervenciones con resultados positivos, las cuales se guían por objetivos, principios o por pautas, esto implica mejorar la relación entre estudiantes y profesores; promoviendo un buen ambiente y convivencia escolar; donde se fomente un aprendizaje activo 
e innovador el cual permite mejorar los procesos de enseñanza-aprendizaje transformando así el sistema escolar y educativo.

En congruencia con esto, mencionaremos algunas de las estrategias utilizadas en la intervención, como es el caso del Armado de siluetas con figuras geométricas: las planificaciones se elaboraron tomando en cuenta la destreza del currículo de educación inicial del MINEDUC (2014e): "identificar figuras geométricas básicas: círculo, cuadrado y triángulo en objetos del entorno y en representaciones gráficas" (pág. 36). Con esta estrategia, se procedió a crear actividades con el uso de las figuras geométricas básicas en GeoGebra, donde se representa el Tangram con diferentes colores; donde los niños y niñas identifican cada figura y la asocian con el color correspondiente. El objetivo de esta actividad se cumplió al clasificar los objetos de formas similares del entorno y reconocer colores primarios y secundarios, como se observa en la figura 1, donde se identifica las figuras geométricas básicas.

Figura 1. Identificación de figuras geométricas básicas; círculo, cuadrado y triangulo.

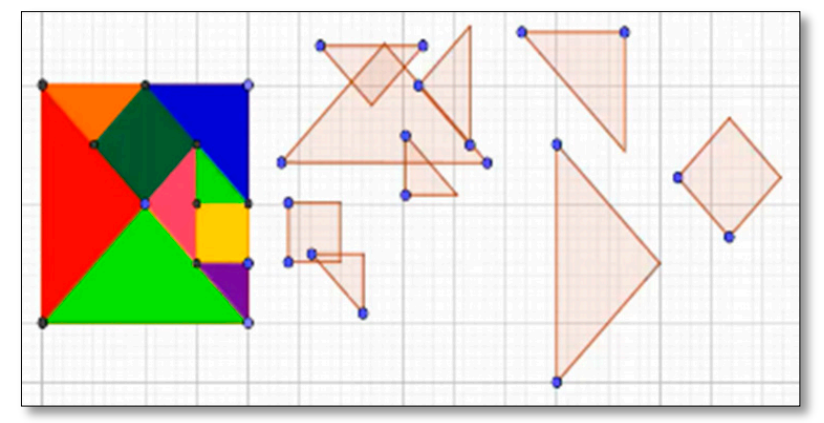

Fuente: Las Autoras (2020).

Así mismo, en las experiencias de aprendizaje se realizó actividades de grande, mediano, pequeño con las figuras geométricas en la aplicación GeoGebra. Esta consistía en que el niño y la niña clasifiquen por tamaños, de acuerdo con el color y a la forma, se observó que no existió dificultad para 
dicha actividad pues, se familiarizaron rápidamente con ella. Además, fue oportuno nombrar los colores y objetos del entorno, llegando a un efectivo reconocimiento, la destreza que se desarrolla en esta actividad conforme al MINEDUC (2014f): es "clasificar objetos con dos atributos (tamaño, color o forma)" (pág. 36), como se observa en la figura 2 con relación a la clasificación de objetos.

Figura 2. Clasificación de objetos (tamaño, color o forma).

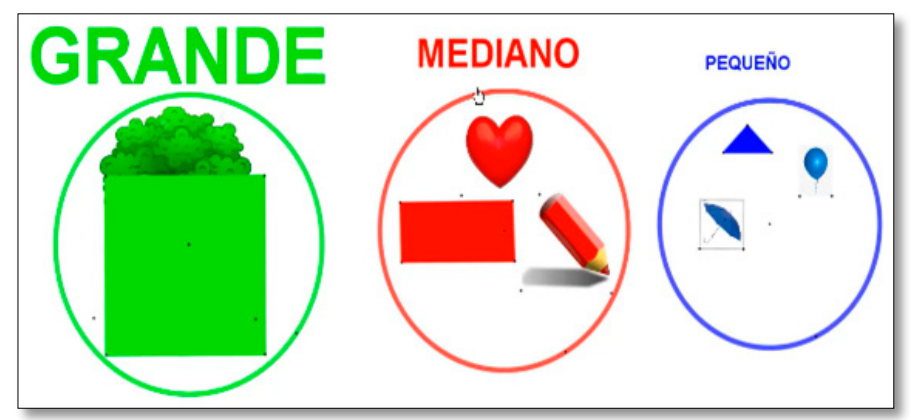

Fuente: Las Autoras (2020).

Con respecto a la relación número cantidad se realizó una planificación para asociar el número con la cantidad correspondiente. Para ello, se procedió a crear una silueta numérica en GeoGebra, esta actividad consistía en que el niño y la niña, primero debían reconocer el número y después asociar a la cantidad correcta.

Figura 3. Relación número cantidad.

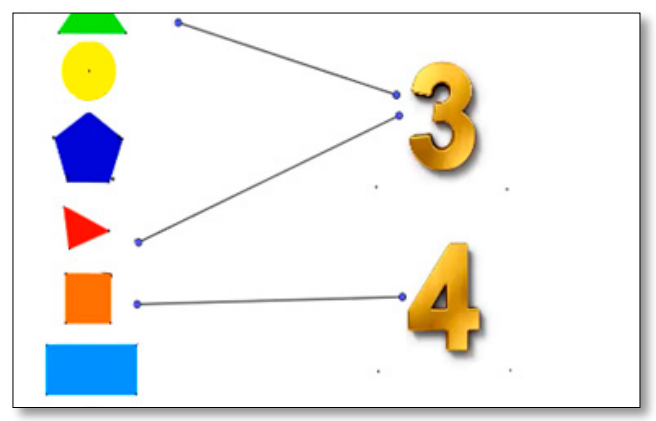

Fuente: Las Autoras (2020). 
De esta forma, para el MINEDUC (2014g): el aprender haciendo mediante el juego desarrolla la destreza de esta actividad que es "Comprender la relación de número-cantidad hasta el 10" (pág. 36). De esta manera, se muestra en la figura 3 con relación número cantidad. De esta manera, se determina que las buenas prácticas son el resultado de un óptimo desempeño, conforme al avance que se obtuvo en cada actividad. Considerando a la propuesta de intervención como un proyecto de continuidad en el proceso de estimulación y adquisición de destrezas, donde se valida que la herramienta de GeoGebra facilita experiencias de aprendizaje de calidad a los niños y niñas del nivel inicial.

\section{Conclusiones}

El refuerzo académico constituye uno de los aspectos relevantes de la presente investigación, pues a partir del diagnóstico de necesidades presentadas por los niños y niñas, se desarrollaron actividades significativas. Como parte de las experiencias de aprendizaje en la consolidación de destrezas y habilidades mediante un cúmulo de las diversas acciones planteadas, con apoyo de una herramienta digital y llevadas al contexto infantil.

El refuerzo académico necesita de aspectos relevantes en su aplicación Siendo, una de las fortalezas el desarrollo del microproyecto de Aproximación a Geometría y Medida y el establecimiento de un cronograma que permite el abordaje de las actividades de manera secuencial y ordenada respondiendo a los objetivos planteados con base en la herramienta de GeoGebra y su aprovechamiento en los procesos de enseñanza-aprendizaje.

Del mismo modo, en el ámbito de relaciones lógico-matemáticas se diseña temáticas de interés con el apoyo de la herramienta de GeoGebra. Que, sin lugar a duda, en la actualidad fortalecen los aprendizajes de los niños y niñas, que se encuentran limitados, por la modalidad virtual en la que se educan. Esto constituye una construcción positiva en la asignatura, como una 
base fundamental para el proceso de abstracción de esta en los subniveles posteriores.

Finalmente, se promueve espacios de socialización de las actividades que permitan generar una perspectiva reflexiva sobre el accionar en la práctica docente. Es así que, como fase final de la intervención se crea un espacio para compartir los resultados obtenidos con los miembros de la comunidad educativa, mismo que permite sistematizar y evaluar las acciones desarrolladas.

En este proceso de crítica constructiva, se destaca que las actividades aplicadas se consideran como buenas prácticas, siendo que, en el campo de la Educación Inicial, se estima que las experiencias se registren con éxito durante su implementación. Sin embargo, como todo proceso en mejora continua deja sugerencias, que serán tomadas en cuenta para ejecutarlas en las propuestas de ciclos académicos posteriores, que inciten a la permanente innovación docente, con base en las buenas prácticas.

\section{Referencias}

Cardoso, E., \& Cerecedo, M. (2008). El desarrollo de las competencias matemáticas en la primera infancia. Revista Iberoamericana de Educación, 47(5), 1-11, e-ISSN: 1681-5653. Recuperado de: https://rieoei.org/RIE/article/view/2270

Cordero, G., Cevallos, D., \& Rivera, K.(coords.). (2019). Buenas prácticas en formación inicial del profesorado. ISBN: 978-84-09-09621-3. España: Institut de Creativitat i Innovacions Educatives de la Universitat de València.

Córdova, P., \& Barrera, H. (2019). Refuerzo académico y la consolidación de aprendizajes de matemática en estudiantes de básica media. Revista Boletín Redipe, 8(11), 100-110, e-ISSN: 2256-1536. Recuperado de: https://doi.org/10.36260/rbr.v8i11.853 
Edel, R. (2003). El rendimiento académico: concepto, investigación y desarrollo. REICE: Revista Iberoamericana sobre Calidad, Eficacia y Cambio en Educación, 1(2), 1-15, e-ISSN: 1696-4713. Recuperado de: https://www.redalyc.org/articulo.oa?id=55110208

Flick, U. (2015). El diseño de Investigación Cualitativa. ISBN: 978-84-7112807-2. Madrid, España: Ediciones Morata, S.L.

Jorquera, M. (2017). Las buenas prácticas como reflejo del estado de la educación musical una revisión bibliográfica. Revista Internacional de Educación Musical, (5), 71-83, e-ISSN: 2307-4841. Recuperado de: https://dialnet.unirioja.es/servlet/articulo?codigo $=6255058$

León, J., \& Cisneros, P. (2021). Competencias y recursos digitales para la enseñanza aprendizaje en educación básica superior. Revista Scientific, 6(20), 92-112, e-ISSN: 2542-2987. Recuperado de: https://doi.org/10.29394/Scientific.issn.2542-2987.2021.6.20.5.92-112

Longás, J., Civís, M., \& Riera, J. (2013). Refuerzo escolar e inclusión educativa: propuesta teórico-práctica a partir de la experiencia de apoyo al éxito escolar del programa CaixaProinfancia. Revista de Educación Inclusiva, 6(2), 106-124, e-ISSN: 1889-4208. Recuperado de: https://dialnet.unirioja.es/servlet/articulo?codigo=4335832

MINEDUC (2014a,b,c,d,e,f,g). Currículo de Educación Inicial. Quito, Ecuador: Ministerio de Educación del Ecuador.

Herberth, A. (2015). EI Refuerzo Educativo. 1ª edición, ISBN: 978-99923-4744-7. San Salvador, El Salvador: UFG Editores.

Ortega, E., \& Taquez, A. (2019). Plan de refuerzo académico para el desarrollo de destrezas con criterio de desempeño. Trabajo de titulación. Azogues, Ecuador: Universidad Nacional de Educación.

Padilla, I. (2018). El juego y la inteligencia lógico-matemática de estudiantes con capacidades excepcionales. Educación y Humanismo, 20(35), 166-183, e-ISSN: 0124-2121. Recuperado de: 
https://dialnet.unirioja.es/servlet/articulo?codigo $=6510626$

Piaget, J. (1961). La formación del símbolo en el niño. México: Editorial Fondo de Cultura Económica.

Reyes-Vélez, P. (2017). El desarrollo de habilidades lógico matemáticas en la educación. Polo del Conocimiento, 2(4), 198-209, e-ISSN: 2550682X. Recuperado de: https://doi.org/10.23857/pc.v2i4.259

Rizzo, K., \& Costa, V. (2019). Matemática, GeoGebra y fotografía, combinados para motivar la enseñanza y el aprendizaje. $V$ Jornadas de Enseñanza e Investigación Educativa en el campo de las Ciencias Exactas y Naturales. Argentina: Universidad Nacional de La Plata.

Rojas-Bello, R. (2020). Introducción del GeoGebra en el proceso de enseñanza-aprendizaje de Geometría a docentes en formación. RECIE: Revista Caribeña de Investigación Educativa, 4(1), 124-134, eISSN: 2636-2147. Recuperado de:

https://doi.org/10.32541/recie.2020.v4i1.pp124-134

Torres, C. (2014). Estrategia didáctica mediada por el software GeoGebra para fortalecer la enseñanza-aprendizaje de la geometría en estudiantes de $9^{\circ}$ de básica secundaria. Proyecto de Investigación. Barranquilla, Colombia: Universidad de la Costa.

Torres, M. (2010). La enseñanza tradicional de las ciencias versus las nuevas tendencias educativas. Revista Electrónica Educare, XIV(1), 131-142, e-ISSN: 1409-4258. Recuperado de: https://www.redalyc.org/articulo.oa?id=194114419012 


\section{Diana Isabel Rodríguez Rodríguez}

e-mail: diana.rodriguezro@unae.edu.ec

Nacida en Azogues, Ecuador, el 1 de mayo del año 1985.

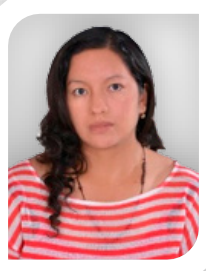

Graduada de Licenciada en Ciencias de la Educación Mención: Matemática por la Universidad Tecnológica Equinoccial de Quito (UTE, 2010), Ecuador; Magíster en Gerencia y Liderazgo Educacional por la Universidad Técnica Particular de Loja (UTPL, 2016), Ecuador; Doctoranda en Ciencias de la Educación en la Universidad Nacional de Cuyo (UNCUYO), Mendoza, Argentina; Investigadora en Pedagogía de la Enseñanza de la Matemática y su interdisciplinaridad en abordaje de temas de interés para la educación actual; Docente Investigadora en la Universidad Nacional de Educación (UNAE). 


\section{Charly Marlene Valarezo Encalada}

e-mail: charly.valarezo@unae.edu.ec

Nacida en Loja, Ecuador el 9 de octubre del año 1982.

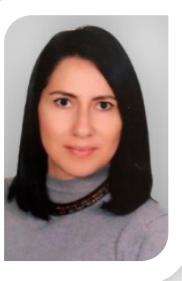
Magister en Educación Infantil de la Universidad Nacional de Loja (UNL), Ecuador; Licenciada en Ciencias de la Educación Especialidad Psicología Infantil y Educación Parvularia de la Universidad Nacional de Loja, Ecuador; Directora del proyecto de vinculación con la comunidad Rincón Infantil de Aprendizaje Móvil Social - RIAMOS; Directora del proyecto de investigación Neopass@ction: Educación Inicial; Miembro del Comité de Redacción de la Runae: Revista científica de investigación educativa (e-ISSN: 2550-6854); Docente investigadora en la Universidad Nacional de Educación (UNAE). 


\section{Dennise Adriana Velecela Cordero \\ e-mail: davelecela@unae.edu.ec}

Nacida en Cuenca Ecuador, el 9 de mayo del año 1999.

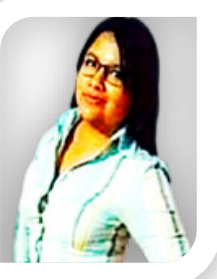

Segunda hija del matrimonio entre Edgar Velecela y Hilda Cordero, mis hermanas Ximena, Jennifer; los estudios primarios los realicé en la escuela Daniel Córdova Toral; los estudios secundarios en el Colegio de Bachillerato Roberto Rodas; me gusta el deporte, me agrada leer, indagar, mi pasión llegar a ser una buena docente. 